\title{
Personalized Gene Expression Analyses of SMAD7 and KLF10 In Breast Cancer
}

\author{
Ali Hosseinzadeh ${ }^{1,2}$, Mohammad Ali Hosseinpour Feizi ${ }^{3}$, Hossein Samadi Kafil ${ }^{4}$, Nasser \\ Pouladi $^{5}$, Morteza Seifi ${ }^{6}$, Reyhaneh Ravanbakhsh Gavgani ${ }^{3}$, Omid Kheyri Nadergoli ${ }^{1,2}$, Leila \\ Rostamizadeh $^{2}$,Vahid Montazeri' ${ }^{7}$ Ashraf Fakhrjou ${ }^{8}$, Ebrahim Sakhinia ${ }^{1,9 *}$. \\ ${ }^{1}$ Immunology Research Center, Tabriz University of Medical Sciences, Tabriz, Iran. ${ }^{2}$ Department of Biochemistry \\ and Clinical Laboratory, Division of Medical Genetics, Faculty of Medicine, Tabriz University of Medical Sciences, \\ Tabriz, Iran. ${ }^{3}$ Department of Biology, Faculty of Natural Sciences, University of Tabriz, Tabriz, Iran. ${ }^{4}$ Drug applied \\ research center, Faculty of Medicine, Tabriz University of Medical Sciences, Tabriz, Iran. ${ }^{5}$ Department of Cellular \\ and Molecular Biology, Faculty of Science, Azarbaijan Shahid Madani University, Tabriz, Iran. ${ }^{6}$ Department of \\ Medical Genetics, Faculty of Medicine and Dentistry, University of Alberta, Edmonton, AB, Canada. ${ }^{7}$ Department \\ of Thoracic Surgery, Noor-E-Nejat Hospital, Tabriz, Iran. ${ }^{8}$ Department of pathology, Tabriz University of Medical \\ Sciences, Tabriz, Iran. ${ }^{9}$ Connective Tissue Disease Research Center, Division of Medical Genetics, Tabriz Genetic \\ Analysis Center (TGAC), Faculty of Medicine, Tabriz University of Medical Sciences, Tabriz, Iran.
}

\begin{abstract}
Many cancer researchers use gene expression analysis for differentiation between tumor and normal cells for diagnostic, prognostic and therapeutic purposes. Most of studies compare either tumor cell lines by normal cell lines or tumor tissue of affected individuals by normal healthy control tissue. But expression of each special gene is unique in different individuals and also in different tissue of same individual. For this reason, here we compare the gene expression levels of SMAD7 and KLF10 in tumor cells and its adjacent normal tissue of breast cancer patients and compared them. For this purpose, a total of 40 tumor and matched tumor-free margin samples were obtained during surgery. The SMAD7 and KLF10 mRNA expression levels in tumor and marginal samples were examined by realtime quantitative PCR. Results are not concordant with previous studies and comparison of only SMAD7 or KLF10 is not useful for differentiating between tumor and margin cells, but ratio analysis of these two genes, SMAD7/ KLF10, can be indicative than study of one gene alone. We concluded that gene expression analysis of tumor cells with adjacent normal tissue are essential for precise identification and interpretation of cancer alterations and have important implications for the diagnostic and therapeutic management of cancer patients.
\end{abstract}

Key words: KLF10, Personalized gene Expression, Real-time PCR, SMAD7, TGF $\beta$

*Authors for correspondence: esakhinia@yahoo.co.uk 


\section{INTRODUCTION}

Transforming growth factor-beta (TGF $\beta$ ), a member of the TGF $\beta /$ Smad signaling pathways, has been established as essential for diverse effects, ranging from cell growth, differentiation, migration and development of multi-organ systems to immune modulation and apoptosis $(1,2)$. Previous studies have shown that members of this pathway have both proliferative and anti-proliferative properties $(3,4)$. SMAD7 (The inhibitory Smad), has been shown to function as an intracellular antagonist of TGF- $\beta$ signaling (5). In several cell types, SMAD7 expression is induced by TGF- $\beta$ family members, indicating an important role of SMAD7 in feedback regulation of TGF- $\beta$ signaling (6). If this feedback is aberrantly regulated can results disease state. Aberrant expression of SMAD7 can cause uncontrolled activity of TGF- $\beta(7,8)$. Expression of SMAD7 in epithelial tissues is very low, but it is upregulated in human pancreatic cancers (9). Significant expression of SMAD7 is also detected in colorectal cancer (10). SMAD7 is also up- regulated in several other cancers (5). SMAD7 can mediate the crosstalk between TGF- $\beta$ and other signaling pathways and has also important role in pathological processes that can be potential therapeutic role in treating both fibrosis and inflammation (2).

Human KLF10 also known as TIEG1( TGF- $\beta$ inducible early gene-1 ) belongs to the Krüppel-like factor (KLF) family, that its Expression in cancer cells has been shown to inhibit cell proliferation and induce apoptosis $(11,12)$. Furthermore, expression level of KLF10 has been shown to be reversely associated with the breast and pancreatic cancer stages and evolution $(11,13,14)$. Loss of KLF10 gene expression in pancreatic cancers has been shown significantly correlated with tumor cell proliferation, tissue invasion, migration and metastasis (13). Whereas, over-expression of KLF10 was shown to mimic the actions of TGF- $\beta$ that regulate cell proliferation, and induce apoptosis $(4,8,10)$. KLF10 also induce apoptosis via reactive oxygen species formation [15]. KLF10 is a transcription factor, which can bind to Sp1 sites on many gene promoters and regulate their transcription $(16,17)$. For example, KLF10 can repress the transcription of SMAD7 gene by directly binding to its promoter (18). KLF10 by limiting the negative feedback of SMAD7 gene and activation of the Smad2 gene in TGF $\beta$-Smad signaling pathway play an important role in temporarily enhancing the intensity of this pathway $(19,20,21)$.
Given the reverse relationship between the expression levels of KLF10 and SMAD7 genes as described above, here we examine expression of KLF10 and SMAD7 in order to find expression patterns of these two genes that may be use afterward for diagnostic, prognostic or therapeutic targets. Most previews studies compared either tumor cell lines by normal cell lines or tumor tissues of affected individuals by normal healthy control tissues. In this study we compared expression of these two genes in tumor cells and its adjacent normal tissue to finding personalized results, and to understanding whether these results are concordant with previews studies.

\section{MATERIALS AND METHODS}

\section{Study Design}

A descriptive study design was carried out under collaboration between the Noor-E-Nejat Hospital and Tabriz Genetic Analysis Center of Tabriz University of Medical Sciences. The protocol of the study was approved by the Ethical Committee of Tabriz University of Medical Sciences, which was in compliance with the Helsinki Declaration. Written informed consent was obtained from all participants.

\section{Study Population and Sample Preparation}

A total of 40 woman breast tumors and its 40 adjacent normal tissue samples were obtained during surgery. Based on pathological results, all tumors were ductal carcinoma, and all margins were free of tumor cells. All patients underwent appropriate surgery at Noor-E-Nejat Hospital of Tabriz in 2014. All patients were clearly identified as having breast cancer based on the clinic-pathological findings. The median age of patients with breast cancer was 48.47 \pm 12.29 years (range $28-80$ years). All samples were obtained immediately after surgery and fresh tumor tissues were snap frozen in liquid nitrogen, and then were carried out to $-70^{\circ} \mathrm{C}$ freezer.

\section{RNA Extraction and cDNA Synthesis}

All samples were subjected to total RNA extraction using TRIZOL (Invitrogen), according to protocol recommended by the manufacturer. The quality and quantity of extracted RNA was analyzed by agarose gel electrophoresis and nanodrop (nd1000). For removing of DNA contamination probability, $2 \mu \mathrm{g}$ of extracted RNA was treated by DNAase1 (Fermentase). First-strand cDNA (complementary DNA) was synthesized from $2 \mu \mathrm{g}$ of total RNA treated by DNAse1 using random hexamer primers and Reverse Transcriptase (Fermentase). For 
determining of cDNA synthesis PCR (polymerase chain reaction) are done by using house-keeping gene primers. For showing nonentity of genomic DNA contamination RT minus control PCR are done, until all samples that are used for quantitative real time PCR been without genomic DNA contamination.

\section{Standards as Gene-Specific Quantity Marker}

A dilution series of human genomic standards for absolute quantification was generated using human genomic DNA (hgDNA). hgDNA (Promega, USA) was homogenized by sonication and serially diluted in Tris-EDTA (TE) buffer to produce standards in which the number of DNA molecules ranged from $1 / 10$ to $1 / 100,000$. Each standard was then placed into aliquots of $1 \mathrm{ml}$ and stored at $-20^{\circ} \mathrm{C}$.

\section{Real-time Quantitative PCR}

Primers for SYBR Green real-time PCR were designed for each gene, details of which are shown in Table 1. For each gene, SYBR Green real-time PCR was performed in $15 \mu \mathrm{l}$ target volume using $4 \mu \mathrm{l}$ of cDNA from each sample, $7.5 \mu$ SYBR Green master mix (Takara, Japan) and 3.5 $\mu$ l of each gene specific forward and reverse primers. All real time PCRs were performed with the following settings: $2 \mathrm{~min}$ at $95^{\circ} \mathrm{C}$, followed by 40 cycles of $95^{\circ} \mathrm{C}$ for $10 \mathrm{~s}, 60^{\circ} \mathrm{C}$ for 60 s. All samples, as well as the five serial human genomic standards, were measured in each gene. Negative controls (NTC) were prepared each time. Heat dissociation (PCR product melting curve) analysis at the end of the PCR was performed for confirmation of single product amplification in each micro-tube.

Table.1 Primers used for PCR

\begin{tabular}{lll}
\hline Gene & \multicolumn{1}{c}{ Reverse primer } & \multicolumn{1}{c}{ Forward primer } \\
\hline KLF10 & CATCAACATCTGCCACTG & CAGCATCCTCAACTATCAG \\
SMAD7 & CATAAACTCGTGGTCATTG & CAGTTACCCCATCTTCATC \\
GAPDH & GGTTGAGCACAGGGTACTTTA & CATGGCCTCCAAGGAGTAAG
\end{tabular}

Values are means \pm SD

\section{Normalization}

Normalization using the housekeeping gene glyceraldehyde-3-phosphate-dehydrogenase

(GAPDH) was used in order to identify the expression levels of the KLF10 and SMAD7 genes. The expression levels of the housekeeping and the KLF10 and SMAD7 genes were measured by realtime quantitative PCR. The mean cycle threshold $(\mathrm{Ct})$ values and the copy number of each gene were determined. The mean copy number of the housekeeping gene was calculated and then divided by the highest housekeeping gene in all samples, resulting in a normalization correction factor. Following real-time PCR amplification and quantification of the selected genes, this factor was used for the normalization of the expression levels for the KLF10 and SMAD7 genes measured. The standard curves, which were plotted, demonstrated the ability of the method to accurately measure the expression levels of KLF10 and SMAD7 genes.

\section{Statistical analysis}

Initial analysis indicated that the data were not normally distributed; therefore, nonparametric tests were used. Spearman correlation coefficient was used. Also, statistical analysis was performed in each of the diagnostic groups using Mann-Whitney $\mathrm{U}$ with $\mathrm{p} \leq 0.05$ deemed as statistically significant.
All analyzes were performed using SPSS v.19 software (SPSS for Windows, Chicago, IL).

\section{RESULTS}

\section{Real-time PCR Amplification Plot, Standard Curve and Heat Dissociation (PCR Product Melting Curve) Analysis}

For each gene, real-time PCR was applied. Negative template controls (NTC) and human genomic standards were prepared each time. Standard curves were plotted using $\mathrm{Ct}$ values of the fivefold serial dilutions of the human genomic DNA for the optimization and efficiency of real-time PCR reactions. Heat dissociation (PCR product melting curve) analysis at the end of the PCR confirmed amplification of single product in each micro-tube and showed that no dimers and unspecific products interfere with the reaction (Figure.1). 


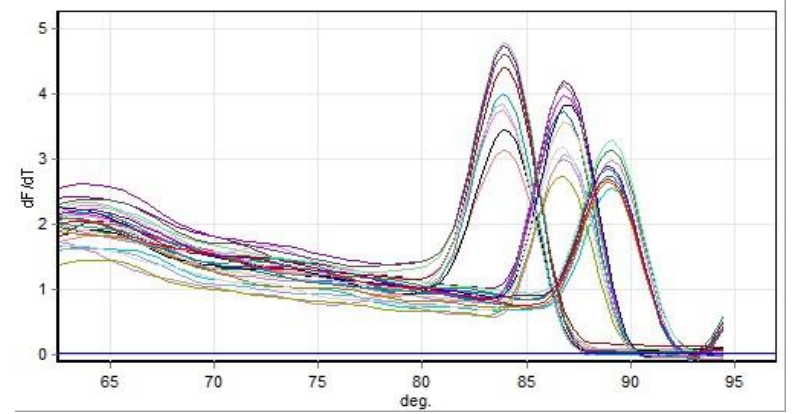

Curves from left to right: KLF10, SMAD7, GAPDH

Fig.1. Melt curve analysis of real time PCR

\section{Comparison of KLF10 Gene Expression Level in Tumor and Margin}

Our results showed that KLF10 expression level in marginal cells is higher than tumor cells $(62.5 \%)$. At the rest $37.5 \%$ of cases KLF10 expression in tumor cells was higher than its marginal cells. Ratio = (conc TKLF10/conc TGAPDH)/(conc MKLF10/ conc MGAPDH)(Figure.2). Mann-Whitney U test show no statistical significance.

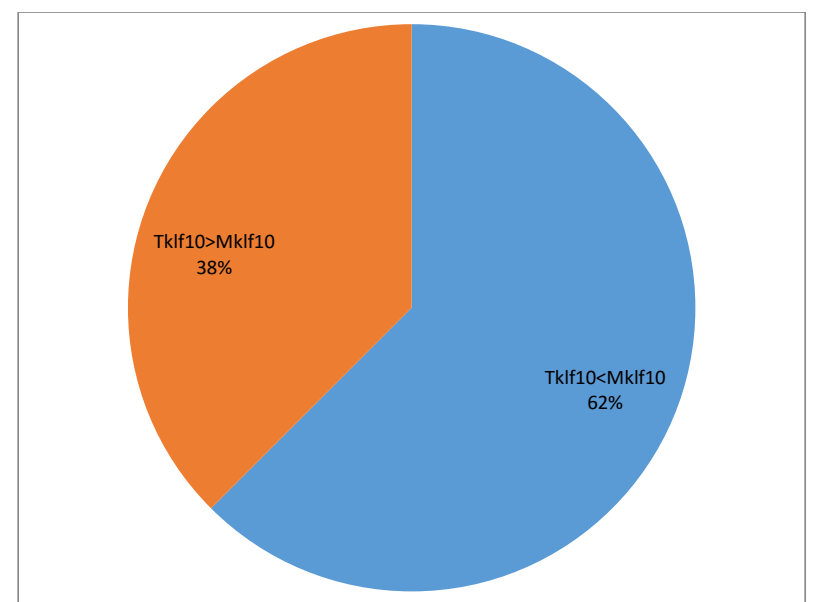

Fig.2. Comparison of klf10 gene in tumor and margin

Comparison of SMAD7 Gene Expression Level in Tumor and Margin

Our results showed that SMAD7 expression level in tumor cells is higher than marginal cells $(62.5 \%)$. At the rest of $37.5 \%$ of cases SMAD7 expression level in marginal cells was higher than its tumor cells. Ratio = (conc TSMAD7/conc TGAPDH $) /$ (conc MSMAD7/conc MGAPDH) (Figure.3). MannWhitney U test are showed no statistical significance.

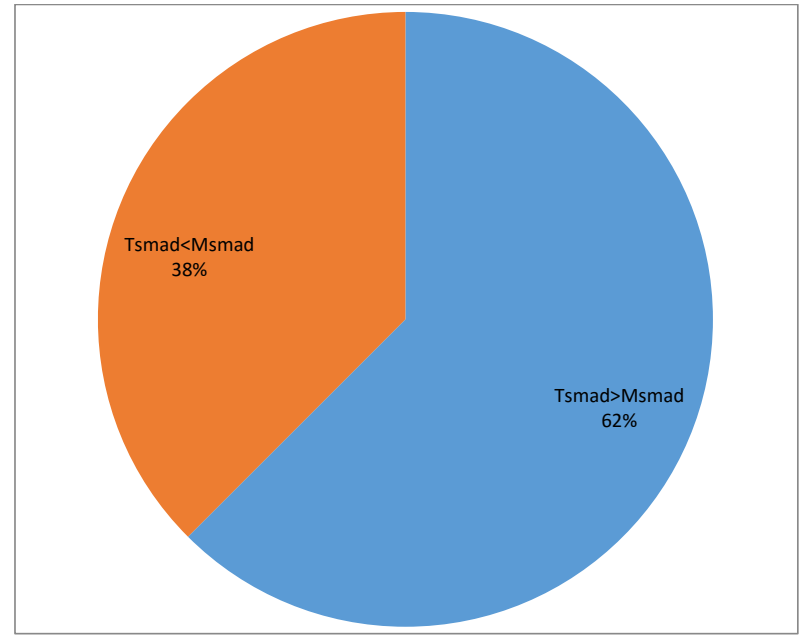

Fig.3. Comparison of smad7 gene in tumor and margin

\section{Comparison of SMAD7/ KLF10 Ratio in Tumor and Margin}

We also calculated the proportion of (tumor SMAD7/tumor KLF10) / (margin SMAD7/margin KLF10):

Ratio = (conc TSMAD7/conc TKLF10)/ (conc MSMAD7/conc MKLF10)

Our results showed that this proportion at $75 \%$ of cases in tumor cells is higher than its adjacent normal tissue (Figure.4).The significance of this proportion is that in this way we don't need to house-keeping gene for normalization between tumor and margin samples. In other calculation methods that uses housekeeping genes for normalization, because housekeeping genes may change in tumor cells, can induce false calculation results. For this reason the use of this proportion can remove the need for housekeeping genes for normalization between two samples and can accurate calculation results. MannWhitney U test was performed, and results show statistical significance $(\mathrm{p}=0.004)$. 


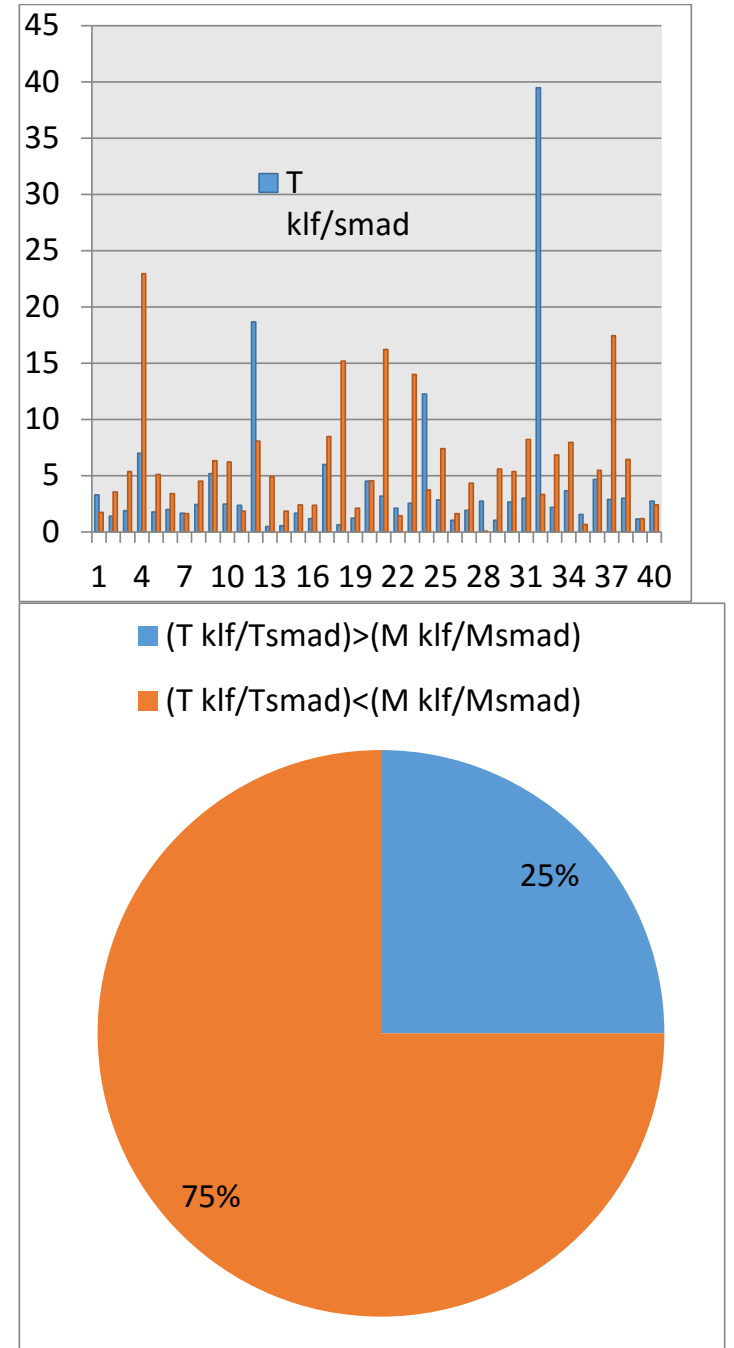

Fig.4.comparison of smad7/klf10 ratio in tumor and margin

\section{Clinical Correlation}

No correlation was identified between SMAD7 and the progression of breast tumor stages, between KLF10 and progression of breast tumor stages, and also between these genes and grade of tumors in our patients. The SMAD7 gene had significant positive correlation by KLF10 in tumor cells (Figure.5), and weak positive correlation in margin cells.

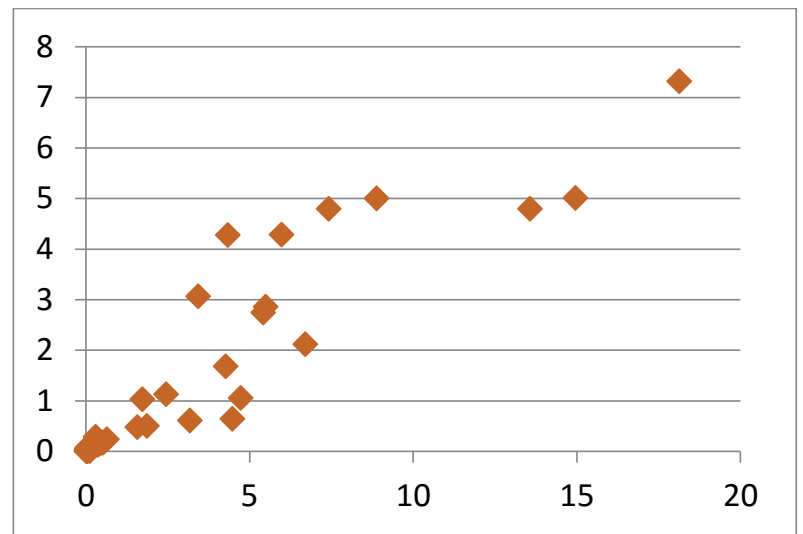

Fig.5. Correlation between expression levels of SMAD7 and KLF10 in tumor cells.

\section{DISCUSSION}

Our results from comparison of KLF10 expression levels between tumor and margin samples showed that this gene was decreased in $62.5 \%$ of cases in tumor cells, but these results were not statistically significant. Besides, in $37.5 \%$ of cases KLF10 decreased in margin cells. Also, SMAD7 in $62.5 \%$ of cases in tumor cells have increased. This low level of differences indicate that use of only KLF10 or SMAD7 may not differentiate between tumor and marginal cells and were not important for diagnosis and treatment of breast tumors. According to previous studies that have been reported increase in expression level of SMAD7 $(5,6)$, and decrease in expression level of $\operatorname{KLF} 10(11,16)$ can cause feedback regulation of TGF- $\beta$ signaling and cause cell proliferation and inhibit apoptosis, and cause tumor formation.Most of these studies are investigated either by comparing of tumor cell lines by normal cell lines or tumor tissue biopsy samples by normal tissue biopsy of healthy control individuals. These comparisons can't be accurate because expression of each special gene is unique in different individuals and also in different tissue of same individual. According to this fact that for successful therapy in personalized medicine, analysis of DNA mutations should be done on both tumor cells and other cells of body (blood or salvia cells, for example) for excluding germ line mutations from somatic mutations (22).Expression analysis also should be conducted in tumor with normal samples of same tissue of same individual. Also, tumor only expression analysis will expose by the same problems. Targeting of one gene that its expression in tumor analysis (without analyzing normal cells of the same tissue) show increase, can disrupt more 
normal cells than cancer cells, because cancer cells are more resistant to therapy than normal cells. Also we applied the ratio analysis (SMAD7/ KLF10) for differentiation between tumor and margin cells. This ratio in $75 \%$ of cases in tumor cells was higher than margin cells, and was statistically significant. In this ratio we used two genes with antagonist function that improved differentiation of two related samples. According to this fact that any housekeeping genes may change in cancer cells (23-25) and cause normalization achieve inaccurate results, ratio analysis can remove this problem and improve differentiation and diagnostic results. Ratio analysis also can be useful for surgeons in differentiation of tumor from margin and reducing of surgical error in tumor removal, tumor clearance, nonrecurring and the success of surgery. Thus, gene expression analysis should be done on more than one gene and the best is that expression evaluation is done on total genes of one special signaling pathway in both tumor and margin, because in different cell types of different individuals, decrease or increase of special genes can be occur in equilibrium with increase or decrease of its antagonist gene, and this changes can't be the main cause of tumor formation. Therefore targeting of one special gene without experiment of other genes involved in special signaling pathway may impair total signaling pathway in normal cells.

Reinholz et al. reported that progression and development of breast tumors are associated with decrease and increase in KLF10 and SMAD7 gene expression levels, respectively, and these findings can be used in molecular staging of breast tumors (3), however, our results didn't show a potential correlation between the increase of SMAD7 and decrease of KLF10 with progression of breast tumors stages. Indeed, our findings showed that SMAD7 has significant positive correlation with KLF10 in tumor cells, and weak positive correlation in margin cells. Tumor grades also did not show correlation with SMAD7 or KLF10 expression levels. These findings showed that SMAD7 and KLF10 gene expression analysis may not help for molecular staging of breast tumors. Molecular staging by expression analysis can be accurate at the time that expression of genes involved in staging, following stable rules and no change from patient to patient. But each tumor has unique changes and heterogeneity is a main characteristic of many cancers (26-29) and expression of genes can vary from patient to patient.

\section{CONCLUSION}

Our studies suggest that for personalized medicine on the base of gene expression analysis, evaluation of tumor cells should be done with normal cells of the same tissue in cancer patients, because the tumoronly analyses may impair patients by inaccurate administration of cancer therapies. Also, our study suggest that ratio analysis of antagonist genes without using housekeeping gene for differentiation between tumor and margin tissues is more useful than analysis of one gene alone, and can be helpful in tumor surgery. Also, molecular staging of cancers because of heterogeneity and hetero-expressivity of tumors can't be accurate and useful.

\section{ACKNOWLEDGEMENTS}

The authors would like to thank immunology research center of Tabriz University of Medical Sciences for their financial support.

\section{Disclosure of interest}

The authors declare no conflicts of interest.

\section{REFERENCES}

[1] Dowdy SC, Mariani A, Reinholz MM, Keeney GL, Spelsberg TC, Podratz KC, et al. Overexpression of the TGF- $\beta$ antagonist Smad7 in endometrial cancer. Gynecologic Oncology 2005. 96: 368-373.

[2] Yan X, Liu Z, Chen Y. Regulation of TGF- signaling by Smad7. Acta Biochimica Et Biophysica Sinica 2009. 41: 263-272.

[3] Reinholz MM, An M-W, Johnsen SA, Subramaniam M, Suman VJ, Ingle JN, et al. Differential Gene Expression of TGF $\beta$ Inducible Early Gene (TIEG), Smad7, Smad2 and Bard1 in Normal and Malignant Breast Tissue. Breast Cancer Res Treat Breast Cancer Research and Treatment 2004. 86: 75-88.

[4] Pickup M, Novitskiy S, Moses HL. The roles of TGF $\beta$ in the tumour microenvironment. Nature Reviews Cancer Nat Rev Cancer 2013. 13: 788-799.

[5] Halder SK, Beauchamp RD, Datta PK. Smad7 induces tumorigenicity by blocking TGF- $\beta$-induced growth inhibition and apoptosis. Experimental Cell Research 2005 307: 231-246.

[6] Derynck R, Akhurst RJ, Balmain A. TGF-beta signaling in tumor suppression and cancer progression .Nature Genetics Nat. Genet 2001, 29: 117-129.

[7] Dong C, Zhu S, Wang T, Yoon W, Li Z, Alvarez RJ, et al. Deficient Smad7 expression: A putative molecular 
defect in scleroderma. Proceedings of the National Academy of Sciences 2002, 99: 3908-3913.

[8] Nakao A, Okumura K, Ogawa H. Smad7: a new key player in TGF- $\beta$-associated disease. Trends In Molecular Medicine 2002, 8: 361-363.

[9] Kleeff J, Ishiwata T, Maruyama H, Friess H, Truong P, Büchler MW, et al. The TGF- $\beta$ signaling inhibitor Smad7 enhances tumorigenicity in pancreatic cancer. Oncogene 1999, 18: 5363-5372.

[10] Korchynskyi O, Landstrom M MN, Stoika R, Funa K, Heldin C-H, Dijke PT, et al. Expression of Smad proteins in human colorectal cancer. International Journal Of Cancer Int J Cancer 1999, 82: 197-202.

[11] Hwang Y-C, Yang C-H, Lin C-H, Ch'ang H-J, Chang $\mathrm{VH}, \mathrm{Yu}$ WC. Destabilization of KLF10, a tumor suppressor, relies on thr93 phosphorylation and isomerase association. Biochimica Et Biophysica Acta (BBA) - Molecular Cell Research 2013, 1833: 30353045 .

[12] Subramaniam M, Hawse JR, Rajamannan NM, Ingle JN, Spelsberg TC. Functional role of KLF10 in multiple disease processes. BioFactors 2010, 36: 8-18.

[13] Chang VH, Chu P-Y, Peng S-L, Mao T-L, Shan Y-S, Hsu C-F, et al. Krüppel-Like Factor 10 Expression as a Prognostic Indicator for Pancreatic Adenocarcinoma. The American Journal Of Pathology 2002, 181: 423 430.

[14] Johnsen SA, Subramaniam M, Janknecht R, Spelsberg TC. TGF $\beta$ inducible early gene enhances TGF $\beta /$ Smad-dependent transcriptional responses. Oncogene 2002, 21: 5783-5790.

[15] Ribeiro A, Bronk SF, Roberts PJ, Urrutia R, Gores GJ. The transforming growth factor? 1-inducible transcription factor, TIEG1, mediates apoptosis through oxidative stress. Hepatology 1999, 30: 1490-149.

[16] Jin W, Chen B-B, Li J-Y, Zhu H, Huang M, Gu S-M, et al. TIEG1 Inhibits Breast Cancer Invasion and Metastasis by Inhibition of Epidermal Growth Factor Receptor (EGFR) Transcription and the EGFR Signaling Pathway. Molecular and Cellular Biology 2011, 32: 5063.

[17] Jiang L. Transactivation of the TIEG1 confers growth inhibition of transforming growth factor- $\beta$-susceptible hepatocellular carcinoma cells. World Journal of Gastroenterology 2012, 18: 2035.

[18] Johnsen SA, Subramaniam M, Monroe DG, Janknecht R, Spelsberg TC. Modulation of Transforming Growth Factor beta (TGFbeta )/Smad Transcriptional Responses through Targeted Degradation of TGFbeta inducible Early Gene- 1 by Human Seven in Absentia Homologue. Journal Of Biological Chemistry 2002, 277: 30754-30759.
[19] Johnsen SA, Subramaniam M, Janknecht R, Spelsberg TC. TGF $\beta$ inducible early gene enhances TGF $\beta /$ Smad-dependent transcriptional responses. Oncogene 2002, 21: 5783-5790.

[20] Johnsen SA, Subramaniam M, Effenberger KE, Spelsberg TC. The TGF? inducible early gene plays a central role in the anti-proliferative response to TGF? Signal Transduction 2004, 4: 29-35.

[21] Hawse JR, Cicek M, Grygo SB, Bruinsma ES, Rajamannan NM, Wijnen AJV, et al. TIEG1/KLF10 Modulates Runx2 Expression and Activity in Osteoblasts. PLoS ONE 2011, 6: e19429.

[22] Jones S, Anagnostou V, Lytle K, Parpart-Li S, Nesselbush M, Riley DR, et al. Personalized genomic analyses for cancer mutation discovery and interpretation. Science Translational Medicine 2015, 283: 283ra53-283ra53.

[23] Khimani A, Mhashilkar A, Mikulskis A, O'malley M, Liao J, Golenko E, et al. Housekeeping genes in cancer: normalization of array data. BioTechniques Biotech 2005, 38: 739-745.

[24] Ferraro A. Altered primary chromatin structures and their implications in cancer development. Cell Oncol (Dordr). 2016 Mar 23. [Epub ahead of print]

[25] Ding Y, Shen J, Zhang G, Chen X, Wu J, Chen W. CD40 controls CXCR5-induced recruitment of myeloidderived suppressor cells to gastric cancer. Oncotarget. 2015 Nov 17;6(36):38901-11.

[26] Vogelstein B, Papadopoulos N, Velculescu VE, Zhou S, Diaz LA, Kinzler KW. Cancer Genome Landscapes. Science 2013, 6127: 1546-1558.

[27] Aktipis CA, Nesse RM. Evolutionary foundations for cancer biology. Evol Appl Evolutionary Applications 2013, 1: 144-159.

[28] Mansfield AS, Murphy SJ, Peikert T, Yi ES, Vasmatzis G, Wigle DA, Aubry MC. Heterogeneity of Programmed Cell Death Ligand 1 Expression in Multifocal Lung Cancer. Clin Cancer Res. 2015 Dec 14. [Epub ahead of print]

[29] Wang A, Wang HY, Liu Y, Zhao MC, Zhang HJ, Lu ZY, Fang YC, Chen XF, Liu GT. The prognostic value of PD-L1 expression for non-small cell lung cancer patients: a meta-analysis. Eur J Surg Oncol. 2015 Apr;41(4):450-6.

Received: Januare 15, 2015; Accepted: March 02, 2016. 